\title{
Principales resultados de investigación sobre Inteligencia Emocional en Docentes Españoles
}

\author{
Álvaro del Carmen Aguayo-Muela. Universidad de Granada \\ M. Carmen Aguilar-Luzón. Universidad de Granada \\ Recepción: 10 de marzo de 2017 | Aceptado: 27 de marzo de 2017 \\ Correspondencia: M. Carmen Aguilar | Correo-e: maguilarluzon@ugr.es
}

\author{
iD 0000-0001-9689-6283 \\ Citar: Aguayo-Muela, A. y Aguilar-Luzón, M.C. (2017). Principales resultados de investigación \\ sobre Inteligencia Emocional en Docentes Españoles. ReiDoCrea, 6, 170-193.
}

\begin{abstract}
Resumen: En la actualidad, el desarrollo de la inteligencia emocional (IE) cobra una gran importancia dentro de la formación integral de la persona, por ello es fundamental desarrollarlo dentro de nuestro sistema educativo de manera significativa. Los profesores se deben convertir en educadores emocionales y para ello es necesario desarrollar dentro de su formación inicial, programas emocionales. Esta investigación se basa en la revisión de 16 estudios realizados con muestras docentes en España, en los que se mide la IE, se correlaciona con otros parámetros educativos y se comprueban la eficacia de programas emocionales. Los resultados indican que a nivel general, la inteligencia emocional de los profesores es "adecuada", que los programas en entrenamiento en IE "aumentan" la inteligencia emocional y que el TMMS-24 es la escala más utilizada en el contexto español. Además se confirman las relaciones positivas entre la IE y el afecto emocional, el burnout y el optimismo. A partir de los resultados obtenidos se hace necesario implantar dentro de la formación del profesorado, programas de entrenamiento en inteligencia emocional para que mejore la calidad de vida, no sólo de los docentes, sino también de los alumnos, y por consiguiente mejore los resultados y la calidad educativa.
\end{abstract}

Palabras clave: Inteligencia Emocional | Docentes Españoles

\section{Main Research Results on Emotional Intelligence in Spanish Teachers}

\begin{abstract}
Nowadays, the development of emotional intelligence (EI) has gained great importance in people's personal growth. Thus, it is essential to promote it within our educational system in a significant manner. Teachers must become emotional educators. For that, it is important to develop emotional programs as part of their initial training. This investigation is based on a review of 16 studies, conducted with samples of teachers in Spain, which measures El, correlates these measures with other educational parameters and confirms the efficacy of emotional programs. The results indicate on a general level: The emotional intelligence of professors is "appropriate"; training programs in El increase emotional intelligence; and TMMS-24 is the scale most used in Spain. A positive relationship between El, emotional affect, burnout and optimism is also confirmed. Based on the results obtained, it is necessary to introduce training programs in emotional intelligence into faculty training to improve the quality of lifestyle of both teachers and students, and consequently improve results and the quality of education.
\end{abstract}

Keywords: Emotional Intelligence | Spanish Teachers

\section{Introducción}

El estudio de la inteligencia emocional (IE), se inicia aproximadamente hace 30 años cuando un psicólogo y profesor de la Universidad de Harvard, Howard Gardner, propuso una nueva visión de las emociones, centrada en una recopilación de aspectos intelectuales. Dos de estos aspectos, denominados inteligencia intrapersonal e inteligencia interpersonal, fueron definidos por Salovey y Mayer (1990) como Inteligencia Emocional. Estos autores, la describieron como "la habilidad para dirigir los propios sentimientos-emociones y la de los demás; saber discriminar entre ellos, y usar esta información para guiar el pensamiento y la propia acción” (pp. 189). 
Actualmente, la IE se define desde diversos planteamientos teóricos, a partir de los que se han generado distintas definiciones e instrumentos de medida (Matthews, Zeidner y Roberts, 2002). Así, la IE puede entenderse como la capacidad de percibir, entender, razonar, modificar y manejar las emociones de uno mismo y la de los demás, discriminar entre ellas y usar dicha información para guiar nuestros pensamientos y comportamientos (Bar-On, 2000; Cherniss y Adler, 2000; Goleman, 1995-1999; Mayer, Caruso y Salovey, 2000; Salovey y Mayer, 1990). El concepto de inteligencia emocional, se encuentra muy relacionado con aspectos de la personalidad: "las personas con cierto tipo de personalidad desarrollarán con más o menos facilidad, con mayor o menor rapidez, sus habilidades emocionales. Al fin y al cabo, la persona no es la suma de sus partes, sino una fusión que convive milagrosamente- de forma integrada" (Fernández-Berrocal y Extremera 2002. p.3).

Diversas investigaciones ponen de manifiesto que en general, un buen desarrollo de la IE presenta efectos beneficiosos sobre diferentes áreas del desarrollo personal (Rey y Extremera, 2011). En este sentido, se ha encontrado que altos niveles de IE se relacionan positivamente con el optimismo (Extremera, Durán y Rey, 2007), con la salud (Extremera y Fernández-Berrocal, 2006), la autoestima (Shutte, Malouff, Hollander y McKenley, 2002) o la adaptación (Boyatzis, Goleman y Rhee, 2000). Por otro lado, bajos niveles de IE están relacionados con el consumo de alcohol y drogas, y una elevada tasa de conductas conflictivas (Mayer, Roberts y Barsade, 2008, cit. Rey y Extremera, 2011) y con el síndrome de estar quemado o "burnout" (Aluja, Blanch y Biscarri, 2002).

Desde el ámbito educativo, la inteligencia emocional va cobrando un especial interés ya que son muchos los beneficios que aporta no sólo a los estudiantes, sino a todo los miembros que conforman nuestro sistema educativo. Dentro de la formación de los estudiantes, se ha demostrado que la IE correlaciona positivamente con el rendimiento académico, debido a que influye en el manejo del estrés, el desarrollo de habilidades intrapersonales y la adaptabilidad (Parker, Summerfeldt, Hogan y Majeski, 2004), y que además, ayuda a ajustar los pensamientos negativos y de alta claridad (negativos y positivos) provocando un ajuste emocional e influyendo posteriormente en el rendimiento académico (Extremera y Fernández-Berrocal, 2001). Parker, Summerfeldt, Hogan y Majerki (2004) encontraron una correlación entre IE y rendimiento escolar de 0,41 en un estudio con 667 estudiantes de secundaria de Canadá utilizando el modelo de Bar-On. Por otra parte, Clavero Muñoz (2011) realizó una revisión de estudios sobre la IE en relación a la violencia y la convivencia escolar, concluyendo en los siguientes puntos: 1. La IE se correlaciona negativamente con la justificación de las agresiones ante los conflictos en el aula y los niveles elevados de impulsividad (Fernández-Berrocal y Extremera, 2002). 2. La alfabetización emocional está relacionada negativamente con problemas de conducta tales como la agresión y conductas antisociales (Liao, Liau, Teoh y Liao, 2003). 3. La falta de disciplina, las agresiones o la hostilidad hacia el profesor están relacionados negativamente con la IE (Mestre, Guil y Gil-Olarte, 2004). 4. La combinación de una baja autoeficacia emocional, aumento de impulsividad y falta de habilidades sociales provocan en los alumnos comportamientos antisociales (Petrides, Frederickson y Furnham, 2004). Se dan relaciones positivas entre la IE y el comportamiento prosocial, pero sin embargo no correlaciona con una conducta agresiva (Gil-Olarte, Palomera y Brackett, 2006). 6. Se encuentra una relación inversamente proporcional entre la inteligencia emocional y los problemas de conducta (Siu, 2009). 7. Un déficit de inteligencia emocional puede estar relacionado con problemas de conducta en adolescentes (Mattingly, 2010).

Para que el estudiante aprenda y desarrolle las habilidades emocionales y afectivas relacionadas con el uso inteligente de sus emociones necesita de un "educador emocional", es decir, un docente que tenga equilibradas sus propias emociones. Una 
buena gestión emocional de los profesores, desarrollará un clima de clase adecuado otorgando a los estudiantes una mayor participación e interés de sus emociones (Sutton y Wealthy, 2003). Sin embargo, el desarrollo de la IE por parte del docente en el aula no debe enfocarse en una sola dirección (profesor-estudiante), sino que la IE debe ser una herramienta en la vida personal del docente. Mearns y Cain (2003) afirman que los profesores que se perciben así mismos como poseedores de habilidades emocionales utilizan más estrategias activas para enfrentarse a las situaciones estresantes del entorno académico (Mestre, Guil, Lopes, Salovey y GilOlarte, 2006).

Diferentes estudios se han centrado en la medición de la inteligencia emocional de estos profesionales, correlacionándolos con otros constructos: "Pensamientos positivos y negativos (Augusto-Landa, López-Zafra y Pulido-Martos, 2011), "Apoyo social" (Rey y Extremera, 2011; Cabello, Fernández-Berrocal, Ruiz-Aranda y Extremera, 2005), Síndrome de estar quemado o "Burnout" (Extremera, Durán y Rey, 2010), "autoeficacia" docente (Perándones y Castejón, 2006), "compromiso" (PenaGarrido y Extremera 2012), o con "la satisfacción vital" (Augusto-Landa, López-Zafra, Martínez de Antoñana y Pulido 2006), obteniendo una correlación positiva en la mayoría de éstos. En este sentido, Sala (2002) observó que la IE incide en los valores, actitudes, creencias y estilos educativos de los propios docentes.

Por ello, parece necesario dotar a nuestros docentes de herramientas específicas sobre inteligencia emocional para incorporar a su desarrollo personal y a su trabajo en el aula. Sin embargo, aun cuando el profesorado se encuentra concienciado en la necesidad de trabajarla en el aula, en la mayoría de las ocasiones, no dispone de la formación adecuada, ni de los medios suficientes para desarrollar esta labor y sus esfuerzos con frecuencia se centran en el diálogo ético-moral, ante el que los estudiantes responden con una actitud pasiva (Abarca, Marzo y Sala 2002). Desafortunadamente, no encontramos dentro del currículum educativo formal, las estrategias y actividades emocionales de forma concreta. Sólo podemos confiar en la personalidad del profesor, y en que éste posea el suficiente control emocional para transmitirlo directamente a sus estudiantes (Extremera y Fernández-Berrocal, 2004).

En definitiva, como apuntan Valles y Valles (2003) la educación emocional debería estar inserta en las distintas áreas curriculares, no en cuanto a su enseñanza/aprendizaje como contenido de cada área, sino cómo estilo educativo del docente que debe transmitir modelos emocionales adecuados en los momentos en los que profesor y estudiantes conviven en el aula.

Por tanto, la necesidad de introducir programas de inteligencia emocional en la formación inicial de los docentes, se convierte en un aspecto primordial para conseguir una mejora en la calidad de la enseñanza (Palomera, Fernández-Berrocal y Brackett, 2008). No obstante, son pocos los programas que han demostrado su eficacia para tal objetivo (Palomera y Gil-Olarte, 2006) y se llevan a cabo mediante un entorno adecuado para la expresión de emociones y exposición a experiencias emocionales distintas dentro del aula (Mayer y Cobb, 2000; Mayer y Salovey, 1997).

En los últimos años, han surgido programas de IE para desarrollar en el ámbito educativo, una competencia que como se ha contextualizado anteriormente, facilita y crea un buen desarrollo académico y profesional de la enseñanza. Entre los diferentes programas podemos encontrar dentro de nuestro país, "la educación responsable" (Fundación Botín), "Actuación integral con políticas públicas de promoción de la IE" (Guipúzcoa, España), "Actividades de investigación y formación en la educación emocional" (GROUP, Cataluña, España), "Programas de Inteligencia Emocional" 
(Laboratorio de Emociones, Málaga, España). Todos ellos trabajan inteligencia emocional en los profesores, dotándoles de herramientas de intervención aplicadas (Cabello, Ruiz-Aranda y Fernández Berrocal, 2010).

\section{Modelos y evaluación de IE}

Entre las diferentes conceptualizaciones de la IE, podemos encontrar los denominados "Modelos mixtos", entendido como la combinación de habilidades mentales con rasgos estables de comportamiento y variables de la personalidad. Incluyen rasgos de personalidad como la motivación, manejo del estrés, asertividad, y ansiedad. Entre ellos destacar: Modelo de Goleman (cociente emocional que complementa al cociente intelectual) y Modelo de Bar-On (intrapersonal, interpersonal, adaptabilidad, manejo del estrés y estado de ánimo en general). Los "Modelos de habilidades", centrados en habilidades mentales que permiten utilizar la información que nos proporciona las emociones para mejorar el procesamiento cognitivo (este modelo es el más utilizado en España). Dentro de este tipo de modelos, podemos citar el modelo planteado por Salovey y Mayer (1990), centrado en la percepción, facilitación, comprensión, dirección y regulación emocional. Además, podemos encontrar "Otros modelos" que incluyen modelos de personalidad como por ejemplo los Modelo de Cooper y Sawaf (1997), Modelo de Boccardo, Sasia y Fontenla (1999) o el de Vallés y Vallés (1999) (García-Fernández y Giménez-Mas, 2010; Fernández-Berrocal, Berrios-Martos, Extremera y Augusto, 2012).

Para evaluar la IE en el ámbito educativo, generalmente, se han utilizado tres formas (Extremera y Fernández-Berrocal, 2002): un "primer grupo", basado en cuestionarios y auto-informes con enunciados verbales y contestados por la propia persona, en una escala de respuesta numérica (principalmente Likert). Un "segundo grupo", formado por medidas de evaluación de observadores externos (consisten en cuestionarios cumplimentados por personas que conviven con el individuo al que se evalúa). $Y$, un "tercer grupo", que reúne las llamadas medidas de habilidad o de ejecución de IE; éstos últimos, son tareas emocionales que la persona a la que se evalúa debe resolver. En concreto en las pruebas de habilidad se combinan ejercicios que permiten inferir la competencia de las personas en la solución de problemas relacionados con las habilidades emocionales, y otros ejercicios que nos proporcionan información sobre el conocimiento que éstas tienen acerca de cómo llevar a cabo las tareas (González Robles, Peñalver González y Bresó Estevez, 2011).

Actualmente, los auto-informes son la medida más utilizada ya que requiere poca inversión económica y menor tiempo de administración, presentan una fácil explicación de las instrucciones y ejecución. Todo esto conlleva a una reducción de fatiga que puede ocasionar otras pruebas de larga duración. Sin embargo, un inconveniente importante que presentan esta forma de medida, las basadas en autoinformes, es la posible distorsión de las respuestas, como consecuencia de las deseabilidad social, razón por la que muchos investigadores prefieren el uso de las medidas de habilidad (González Robles et al. 2011).

Entre los auto-informes más conocidos podemos encontrar el TMMS (Salovey, Mayer, Goldman, Turvey y Palfai, 1995), adaptado al contexto español por FernándezBerrocal y Extremera, (2004), el IE de Schutte (Schutte, Malouff, Hall, Haggerty, Cooper, Golden, y Dornheim, 1998), el EQ-I de Bar-On (1997), y la escala WLEIS (Wong y Law, 2002). Por otro lado, entre las medidas de habilidades más utilizadas, podemos encontrar el MEIS (Mayer, Caruso y Salovey, 1999) y el MSCEIT (Mayer, Salovey y Caruso, 2002; Mayer, et al., 1999), basados en los modelos de Salovey y Mayer, 1990 (Extremera y Fernández-Berrocal, 2003). 
Como podemos observar, son diversos los planteamientos teóricos y las formas de medida de IE que se han utilizado, en los estudios empíricos que han trabajado con muestras de docentes. Esta disparidad de modelos, puede generar resultados diferentes e incluso a veces contradictorios, por lo que creemos necesaria la puesta de marcha de trabajos que permitan unificar los resultados hallados en general, dentro del contexto educativo español, a fin de lograr plasmar un "diagnóstico" del estado actual de esta cuestión: cómo es la IE en los docentes españoles y qué relación tiene con el rendimiento académico. Concretamente, el objetivo de este trabajo ha sido analizar las investigaciones realizadas hasta la fecha sobre IE, que utilizan muestras de docentes españoles, comparando sus resultados y conclusiones de cada estudio.

\section{MÉTODO}

\section{Estrategia de búsqueda}

La estrategia de búsqueda, realizada hasta Septiembre de 2016, se efectuó en cinco bases de datos: ERIC, Web of Science, Scopus, Teseo y Science Direct. Los términos de búsqueda utilizados se basaron en los docentes y sus sinónimos (docente*, maestro*, profesor*, teacher*, professor* ${ }^{*}$ y en la inteligencia emocional y sus conceptos relacionados ("inteligencia emocional", "emotional intelligence", "conciencia emocional", "emotional concience", emociones y emotion), con el fin de recoger todos los estudios potencialmente relevantes para esta revisión. Los sinónimos del mismo término se combinaron con el enlace "OR" y los dos términos entre sí con el enlace "AND" (Benito Peinado et al., 2007). Los términos compuestos por dos palabras, tal y como se recomienda, se entrecomillaron.

\section{Criterios de selección}

Los criterios de selección para identificar los estudios seleccionados fueron: (a) aquellas investigaciones que medían la inteligencia emocional; (b) estudios que utilizaron muestras de docentes españoles de cualquier etapa educativa; (c) estudios que utilizaran diseños de una única medida transversal o diseños de programas de inteligencia emocional aplicados en docentes con medidas pre-post. Además, cualquier aportación científica (libros de congresos, tesis, disertaciones) fue aceptada para su análisis, no restringiéndose a artículos de revista (ver figura 1).

Figura 1. CRITERIOS INCLUSIÓN Y EXCLUSIÓN DE ARTÍCULOS

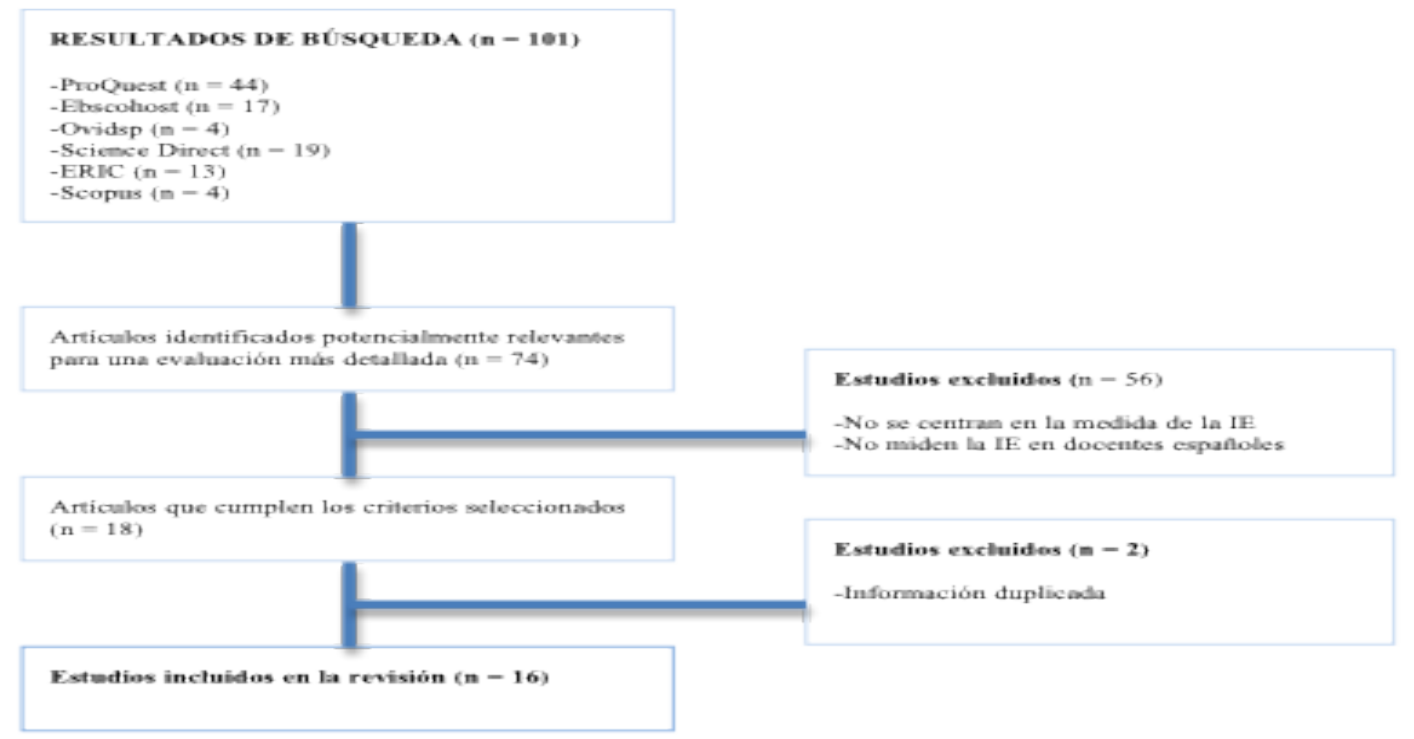




\section{Codificación de los estudios: Análisis de datos}

En todos los estudios seleccionados para esta revisión, se tomaron en consideración los siguientes elementos de codificación: referencia o identificación del estudio; participantes, tomando de ellos las características de género, edad, tipo de centro, nivel o etapa educativa en la que impartían docencia y Comunidad Autónoma de procedencia; instrumentos de medida utilizados de la IE, así como datos de validez y consistencia interna; resultados, en los que se registraron diferenciadamente los factores que componían cada medida de la IE según el instrumento utilizado en la investigación correspondiente; correlaciones de la IE con otras variables. Cabe mencionar que no todos los estudios seleccionados para esta revisión, aportaban la información antes descritas.

\section{Muestra}

A continuación se exponen los estudios que se han seleccionado para el presente artículo (ver tabla 1). Así, en una primera fase de búsqueda se obtuvieron 101 trabajos, de los que se excluyeron 85 por no cumplir los criterios de selección mencionados anteriormente (figura 1). Por tanto, la revisión quedó reducida a 16 artículos. Los estudios de Pérez-Escoda, Filella, Soldevilla y Fondevilla, (2013) y Pérez Escoda, Filella, Alegre y Bizquerra, (2012), al igual que el trabajo de AugustoLanda, López-Zafra, Berrios-Martos y Pulido-Martos, (2012) y de Augusto-Landa, López-Zafra y Pulido Martos (2011), utilizan la misma muestra y resultados en sus diferentes publicaciones, por ello, para evitar la posible pérdida de información, se ha decidido incluir los parámetros que se miden en ambos estudios como si fuera una única investigación. Es preciso comentar que 3 de los 16 estudios (Pérez-Escoda et al., 2013 -2012; De la Fuente, Salvador-Granados y Franco Justo, 2010 y Muñoz de Morales y Bisquerra, 2006) corresponden a programas de Inteligencia Emocional en docentes, por lo que han sido incluidos o clasificados en la categoría sobre la medición de la IE en docentes. Los otros estudios seleccionados son de Muñoz Fernández (2016); Pulido-Martos, López-Zafra, Estévez-López y Augusto-Landa, (2016); Pena Garrido y Extremera, (2012); Rey y Extremera (2011); Pertegal-Felices, CastejónCosta y Martínez (2011); Fernández, Bermejo, Sainz, Llor, Hernández y Soto (2011); Extremera, Durán y Rey (2010); Augusto-Landa, López-Zafra, Martínez de Antoñana y Pulido (2006); Palomera, Gil-Olarte y Brackett (2006); Perándones y Castejón-Costa (2006); Cabello, Fernández-Berrocal, Ruiz-Aranda y Extremera (2005), y Extremera, Fernández-Berrocal y Durán (2003).

TABLA 1. ESTUDIOS SELECCIONADOS

\begin{tabular}{|l|l|l|l|}
\hline AUTORES & AÑO & TíTULO & REFERENCIA \\
\hline $\begin{array}{l}\text { Muñoz } \\
\text { Fernández }\end{array}$ & $(2016)$ & $\begin{array}{l}\text { Inteligencia emocional percibida y su relación con la } \\
\text { satisfacción y el bienestar subjetivo del profesorado. }\end{array}$ & $\begin{array}{l}\text { Inteligencia emocional y bienestar II. } \\
\text { Asociación aragonesa de psicopedagogía. } \\
\text { 10 Edición, 2016. pp. 660-675. }\end{array}$ \\
\hline $\begin{array}{l}\text { Pulido- } \\
\text { Martos et al. }\end{array}$ & $(2016)$ & $\begin{array}{l}\text { The Moderator Role of Perceived Emotional Intelligence } \\
\text { in the Relationship between sources of stress and } \\
\text { Mental Health in Teacher. }\end{array}$ & $\begin{array}{l}\text { The Spanish Journal of Psychology } \\
\text { (2016), 19, e7, pp. 1-10. }\end{array}$ \\
\hline \multirow{2}{*}{$\begin{array}{l}\text { Pérez-Escoda } \\
\text { et al. }\end{array}$} & $(2013)$ & $\begin{array}{l}\text { Evaluación de un programa de educación emocional } \\
\text { para profesorado de primaria * }\end{array}$ & $\begin{array}{l}\text { Educación XXI, 16(1). } \\
\text { pp. 233-254. } \\
\text { ISSN 1139-613X. }\end{array}$ \\
\cline { 2 - 5 } & $(2012)$ & $\begin{array}{l}\text { Developing the emotional competence of teachers and } \\
\text { pupils in school contexts. }\end{array}$ & $\begin{array}{l}\text { Education \& Psychology I+D+i and } \\
\text { Editorial EOS (Spain). }\end{array}$ \\
\hline $\begin{array}{l}\text { Augusto- } \\
\text { Landa et al. }\end{array}$ & $(2012)$ & $\begin{array}{l}\text { Analyzing the relations among perceived emotional } \\
\text { intelligence, affect balance and burnout. }\end{array}$ & $\begin{array}{l}\text { Behavioral Psychology/Psicología } \\
\text { Conductual, Vol. 20, N01, pp. 151-168. }\end{array}$ \\
\cline { 2 - 5 } & $(2011)$ & $\begin{array}{l}\text { Inteligencia Emocional Percibida y estrategias de } \\
\text { afrontamiento al estrés en profesores de enseñanza } \\
\text { primaria: propuesta de un modelo explicativo con } \\
\text { ecuaciones estructurales (SEM). }\end{array}$ & $\begin{array}{l}\text { Revista de Psicología Social, 26(3). } \\
\text { (2011). 413-425. }\end{array}$ \\
\hline & & Inteligencia emocional percibida en profesorado de & \\
\hline
\end{tabular}




\begin{tabular}{|c|c|c|c|}
\hline $\begin{array}{l}\text { Pena-Garrido } \\
\text { et al. }\end{array}$ & (2012) & $\begin{array}{l}\text { primaria y su relación con los niveles de burnout e } \\
\text { ilusión por el trabajo (engagement). }\end{array}$ & $\begin{array}{l}\text { Revista de Educación, 359. Septiembre- } \\
\text { diciembre. }\end{array}$ \\
\hline $\begin{array}{l}\text { Fernández et } \\
\text { al. }\end{array}$ & $(2011)$ & $\begin{array}{l}\text { Percepción socioemocional de los profesores en } \\
\text { adolescentes con altas habilidades versus habilidades } \\
\text { medias. }\end{array}$ & $\begin{array}{l}\text { Revista Electrónica Interuniversitaria de } \\
\text { Formación del Profesorado, 14(3). 55-64. }\end{array}$ \\
\hline Rey et al. & $(2011)$ & $\begin{array}{l}\text { El apoyo social como un mediador de la Inteligencia } \\
\text { Emocional percibida y la satisfacción vital de una } \\
\text { muestra de profesorado. }\end{array}$ & $\begin{array}{l}\text { Revista de Psicología Social, 26(3). 401- } \\
\text { 412. ISSN 0213-4748. }\end{array}$ \\
\hline $\begin{array}{l}\text { Pertegal- } \\
\text { Felices } \\
\text { et al. }\end{array}$ & $(2011)$ & $\begin{array}{l}\text { Competencias socioemocionales en el desarrollo } \\
\text { profesional del maestro. }\end{array}$ & $\begin{array}{l}\text { Educación XXI, 14(2). 237-260. ISSN } \\
\text { 1139-613X. }\end{array}$ \\
\hline $\begin{array}{l}\text { Extremera et } \\
\text { al. }\end{array}$ & $(2010)$ & $\begin{array}{l}\text { Recursos personales, síndrome de estar quemado por } \\
\text { el trabajo y sintomatología asociada al estrés en } \\
\text { docentes de enseñanza primaria y secundaria. }\end{array}$ & $\begin{array}{l}\text { Ansiedad y Estrés, 16(1). 47-60. ISSN } \\
11347937 .\end{array}$ \\
\hline $\begin{array}{l}\text { De la Fuente } \\
\text { et al. }\end{array}$ & $(2010)$ & $\begin{array}{l}\text { Efectos de un programa de entrenamiento en } \\
\text { conciencia plena (Mindfulness) en la autoestima y la } \\
\text { inteligencia emocional percibidas. }{ }^{*}\end{array}$ & $\begin{array}{l}\text { Behavioral Psychology / Psicología } \\
\text { Conductual, } 18 \text { (2). 297-315. }\end{array}$ \\
\hline $\begin{array}{l}\text { Augusto- } \\
\text { Landa et al. }\end{array}$ & $(2006)$ & $\begin{array}{l}\text { Perceived emotional intelligence and life satisfaction } \\
\text { among university teachers. }\end{array}$ & $\begin{array}{l}\text { Psicothema. Vol. 18. 152-157. ISSN } 0214 \\
-9915 .\end{array}$ \\
\hline $\begin{array}{l}\text { Muñoz de } \\
\text { Morales et al. }\end{array}$ & $(2006)$ & $\begin{array}{l}\text { Evaluación de un programa de educación emocional } \\
\text { para la prevención del estrés psicosocial en el contexto } \\
\text { del aula. }{ }^{*}\end{array}$ & $\begin{array}{l}\text { Ansiedad y Estrés 12(2-3). 401-412. ISSN } \\
1134-7937 .\end{array}$ \\
\hline $\begin{array}{l}\text { Palomera et } \\
\text { al. }\end{array}$ & $(2006)$ & $\begin{array}{l}\text { ¿Se perciben con inteligencia emocional los docentes? } \\
\text { Posibles consecuencias sobre la calidad educativa. }\end{array}$ & $\begin{array}{l}\text { Revista de Educación, } 34 . \\
\text { pp. } 687-703 .\end{array}$ \\
\hline $\begin{array}{l}\text { Perandones et } \\
\text { al. }\end{array}$ & $(2006)$ & $\begin{array}{l}\text { Estudio correlacional entre personalidad, inteligencia } \\
\text { emocional y auto eficacia en profesorado de educación } \\
\text { secundaria y bachillerato. }\end{array}$ & $\begin{array}{l}\text { V Congreso Internacional "Educación y } \\
\text { Sociedad". ISBN 84-690-2369-1. }\end{array}$ \\
\hline Cabello et al. & $(2005)$ & $\begin{array}{l}\text { Influencia de la inteligencia emocional y estilos de } \\
\text { respuestas de factores de riesgo psicosocial en } \\
\text { profesores de educación especial. }\end{array}$ & $\begin{array}{l}6^{\circ} \text { Congreso Virtual de Psiquiatría. } \\
\text { Interpsiquis. }\end{array}$ \\
\hline $\begin{array}{l}\text { Extremera et } \\
\text { al. }\end{array}$ & $(2003)$ & Inteligencia emocional y burnout en profesores. & Encuentros en Psicología Social 260-265. \\
\hline
\end{tabular}

$\left(^{\star}\right)$ Pertenecen a estudios que realizan un programa de inteligencia emocional

\section{Resultados}

Los resultados se han estructurado en función de los diferentes instrumentos de medición de IE, resultados obtenidos en docentes, resultados en relación a los programas de IE y finalmente relación con los parámetros de IE y otros constructos. En cada uno de los apartados se comparan los resultados de todas las investigaciones consideradas para este estudio, y se comprueba disparidad de resultados aun utilizando las mismas medidas. A continuación se expone un resumen de los resultados de búsqueda con sus respectivos filtros hasta obtener los 16 estudios seleccionados (figura 1), y un cuadro resumen de cada estudio (ver tabla 2). 
TABLA 2. CUADRO RESUMEN DE LOS ESTUDIOS SELECCIONADOS

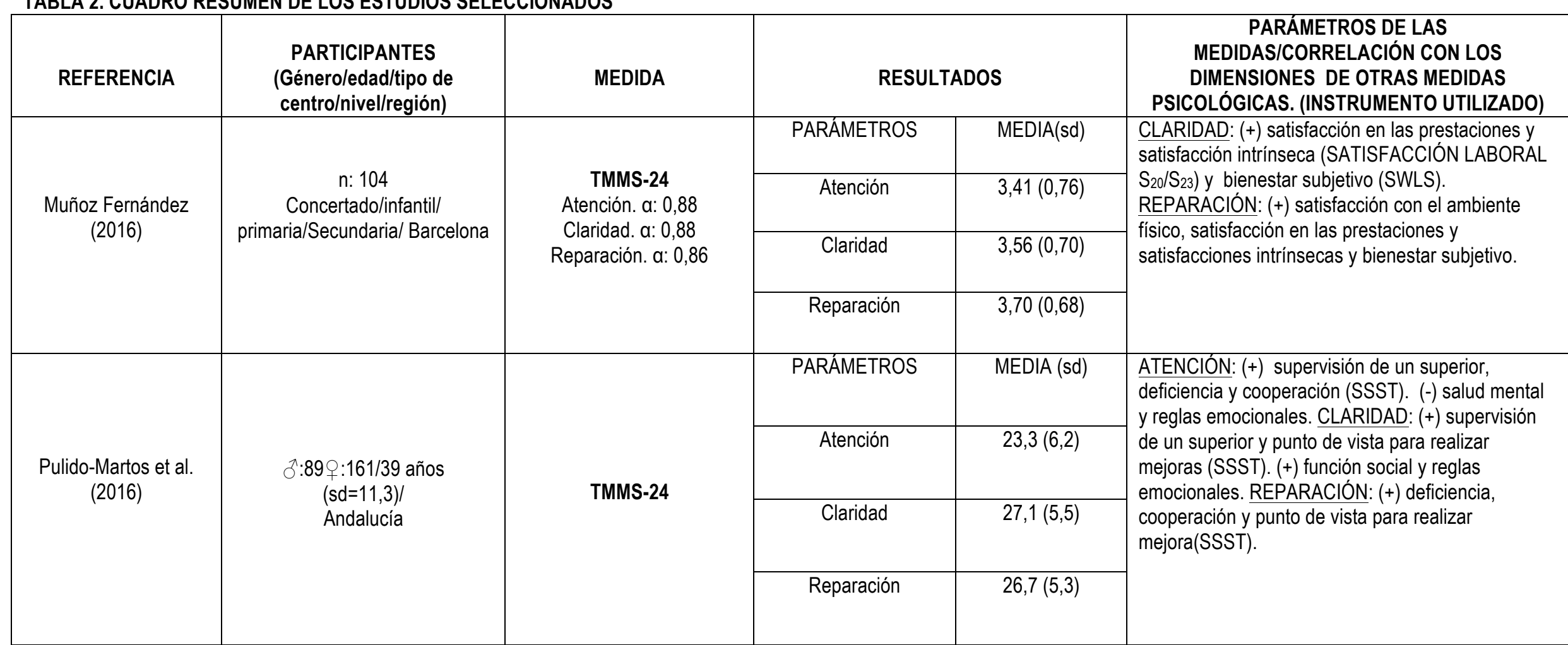

(+): Correlación positiva

(-): Correlación negativa 


\begin{tabular}{|c|c|c|c|c|c|c|c|}
\hline REFERENCIA & $\begin{array}{l}\text { PARTICIPANTES } \\
\text { (Género/edad/tipo de } \\
\text { centro/nivel/región) }\end{array}$ & & \multicolumn{4}{|c|}{ RESULTADOS } & $\begin{array}{c}\text { PARÁMETROS DE LAS MEDIDAS/CORRELACIÓN CON LOS } \\
\text { DIMENSIONES DE OTRAS MEDIDAS PSICOLÓGICAS. (INSTRUMENTO } \\
\text { UTILIZADO) }\end{array}$ \\
\hline \multirow{13}{*}{$\begin{array}{l}\text { Pérez-Escoda } \\
\text { et al. } \\
(2013,2012)\end{array}$} & \multirow{13}{*}{$\begin{array}{c}\mathrm{n}: C^{\lambda}: 110: 81 / \\
\text { Público/Primaria/Cataluña }\end{array}$} & \multirow{13}{*}{$\begin{array}{c}\text { MEDIDA } \\
\text { CDE-A } \\
\text { a: } 0,92 \\
\text { Parámetros a: > } \\
0,69 \\
(2013) \\
\text { CDE-A } \\
\text { a: } 0,91 \\
\text { Cociente E. a: } \\
0,67 \\
\text { Regulación E. a: } \\
0,79 \\
\text { Competencias } S . \\
\text { a: } 0,68 \\
\text { Autonomía E. a: } \\
0,61 \\
\text { Competencias V. } \\
\text { a } 0,72 \\
(2012)\end{array}$} & \multicolumn{2}{|c|}{ PARÁMETROS } & G.E. & G.C. & \\
\hline & & & \multicolumn{2}{|c|}{\begin{tabular}{l|l} 
& Cociente \\
\end{tabular}} & 7,05 & 7,28 & \\
\hline & & & \multicolumn{2}{|r|}{ Regulación } & 5,28 & 5,73 & \\
\hline & & & \multirow[t]{4}{*}{ PRE } & Comp. & 6,05 & 6,08 & \\
\hline & & & & Autonomía & 5,68 & 5,59 & \\
\hline & & & & \multirow{2}{*}{ Comp. Vital } & \begin{tabular}{|l|l|}
7,04 \\
\end{tabular} & 6,89 & - \\
\hline & & & & & 6,12 & \begin{tabular}{|l|}
6,24 \\
\end{tabular} & \\
\hline & & & & Cociente & 7,27 & 7,15 & \\
\hline & & & & Regulación & 5,86 & 5,69 & \\
\hline & & & POS & $\begin{array}{l}\text { Comp. } \\
\text { Social }\end{array}$ & 6,44 & 6,09 & \\
\hline & & & & Autonomía & 5,87 & 5,70 & \\
\hline & & & & Comp. Vital & \begin{tabular}{l|l}
17,35 \\
\end{tabular} & 6,95 & \\
\hline & & & & TOTAL & 6,49 & 6,24 & \\
\hline \multirow{4}{*}{$\begin{array}{l}\text { Augusto-Landa } \\
\text { et al. } \\
\text { (2012 y 2011) }\end{array}$} & \multirow{4}{*}{$\begin{array}{c}\text { n: đ̊:89o:162/39 años } \\
\text { (sd=11,25) } \\
\text { /Público/Primaria/ } \\
\text { Andalucía }\end{array}$} & \multirow{4}{*}{$\begin{array}{c}\text { TMMS-24 } \\
\text { Atención } \\
\text { a: 0,88-0,86 } \\
\text { Claridad } \\
\text { a: 0,90-0,88 } \\
\text { Reparación } \\
\text { a: 0,86-0,8 }\end{array}$} & \multicolumn{2}{|c|}{ PARÁMETROS } & \multicolumn{2}{|c|}{ MEDIA (sd) } & \multirow{4}{*}{$\begin{array}{l}\text { ATENCIÓN Y CLARIDAD: (+) Escala de afecto positivo (PANAS), } \\
\text { Agotamiento emocional, despersonalización y realización (MBI), y } \\
\text { afrontamiento conductual, cognitivo y centrado en la emociones (COPE). } \\
\text { REPARACIÓN: (+) Escala de afecto positivo (PANAS), Agotamiento, } \\
\text { despersonalización y realización (MBI), afrontamiento centrado en las } \\
\text { emociones y escape conductual (COPE). }\end{array}$} \\
\hline & & & \multicolumn{2}{|l|}{ Atención } & \multicolumn{2}{|c|}{$23,30(6,22)$} & \\
\hline & & & \multicolumn{2}{|l|}{ Claridad } & \multicolumn{2}{|c|}{$27,06(5,51)$} & \\
\hline & & & \multicolumn{2}{|l|}{ Reparación } & \multicolumn{2}{|c|}{$26,71(5,32)$} & \\
\hline
\end{tabular}




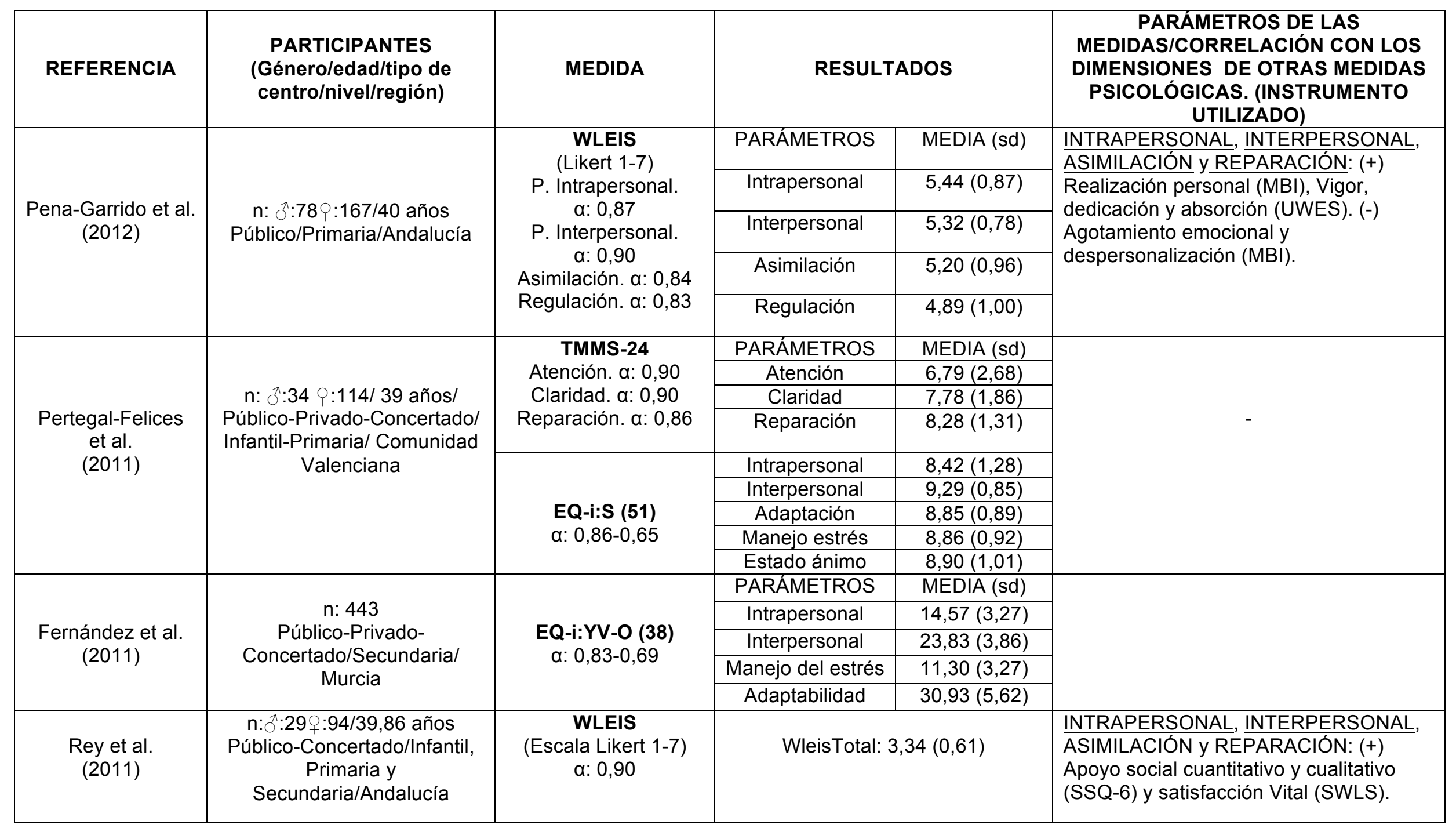




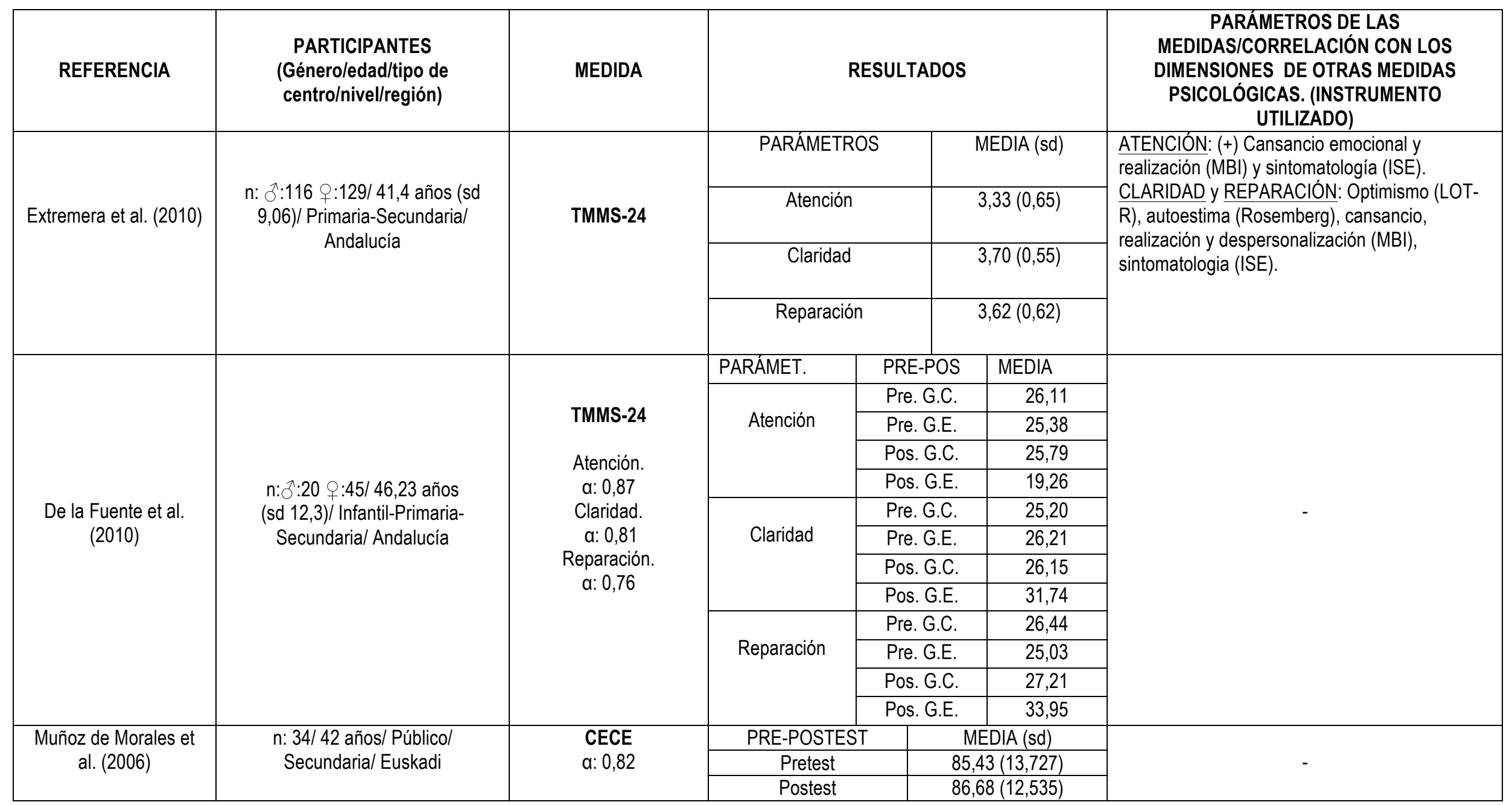




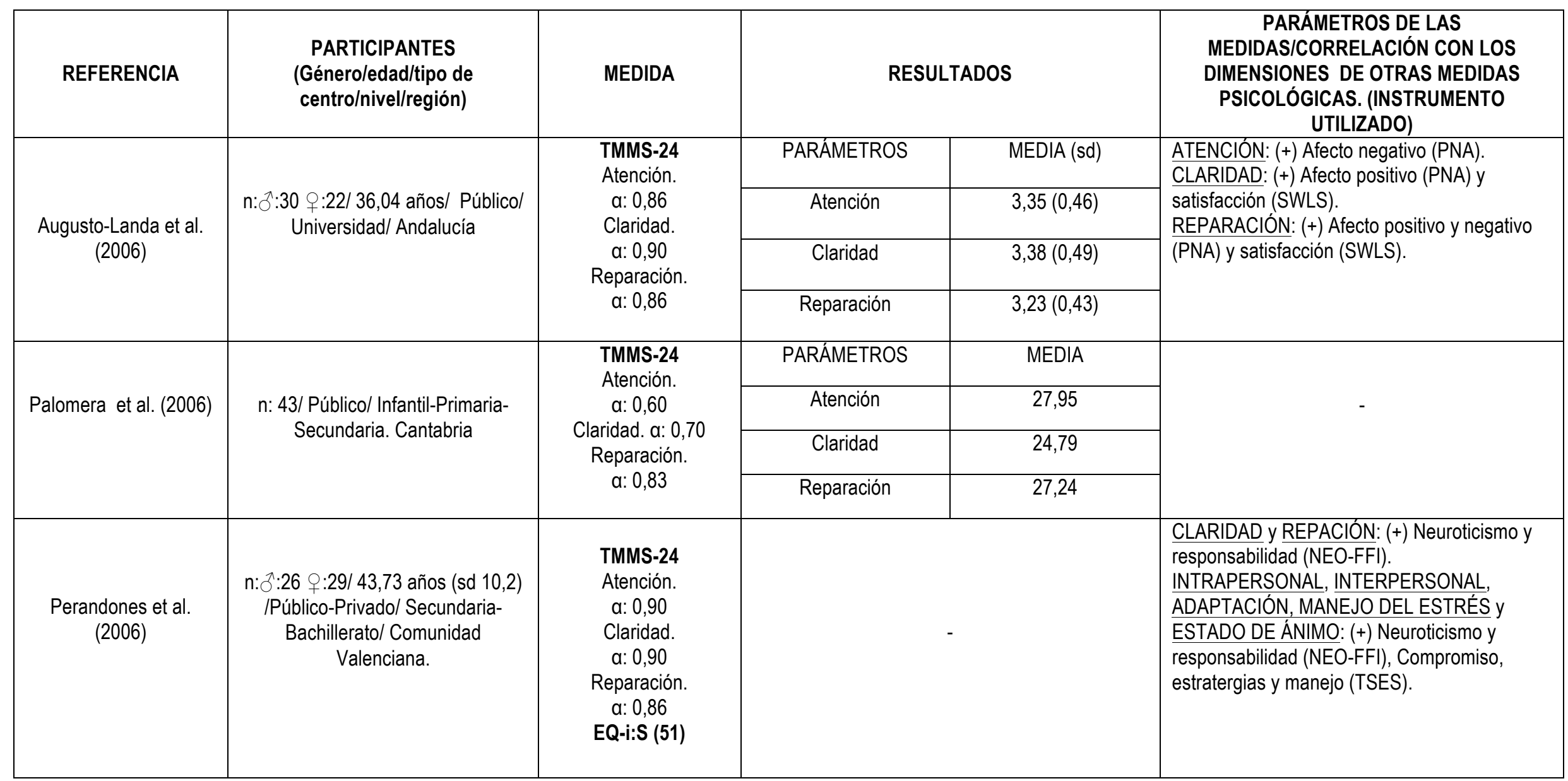




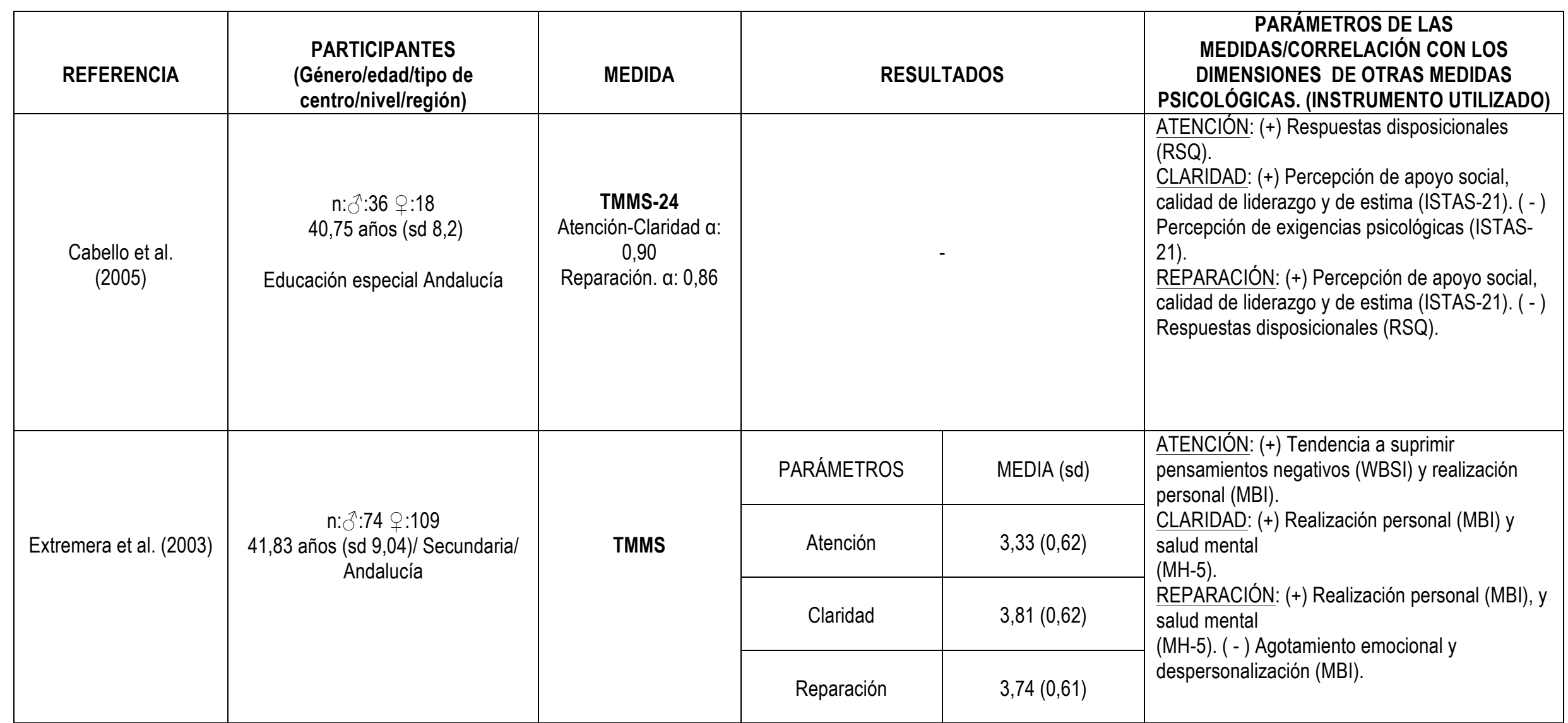




\section{En relación a los instrumentos utilizados}

A continuación se van a exponer los instrumentos de medida de los estudios seleccionados. En todo ellos se realizará una breve descripción, así como la información de su fiabilidad.

\section{Trait Meta-Mood Scale (TMMS)}

Salovey y Mayer diseñaron el Trait Meta-Mood Scale (TMMS), que evalúa el metaconocimiento de los estados emocionales mediante 48 ítems (FernándezBerrocal, Extremera y Ramos, 2004). Existe para este cuestionario una versión adaptada al castellano por Fernández-Berrocal, Alcaide, Domínguez, FernándezMcnally, Ramos y Ravira (1998) con propiedades psicométricas similares a la prueba original (Extremera, Fernández-Berrocal y Durán-Durán, 2003). Posteriormente, ha sido adaptada y validada al contexto español por Fernández-Berrocal y Extremera (2004), quedando reducida a 24 ítems. La escala de respuesta utilizada se trata de una escala tipo Likert con 5 anclajes. EI TMMS-24 mide tres dimensiones: atención emocional (capacidad de atender a los sentimientos de forma adecuada), claridad emocional (comprendo bien mis estados emocionales) y reparación emocional (capacidad de regular los estados emocionales de forma adecuada). A continuación se muestra el coeficiente de fiabilidad $(\alpha)$ que han obtenido los diferentes estudios analizados en el presente trabajo (ver tabla 3 ).

TABLA 3. FIABILIDAD OBTENIDA EN LOS DIFERENTES ESTUDIOS CONSIDERADOS QUE UTILIZAN EL TMMS-24

\begin{tabular}{|c|c|c|}
\hline PARÁMETROS & FIABILIDAD & AUTORES \\
\hline \multirow{6}{*}{ ATENCIÓN } & a: 0,90 & Pertegal et al. (2011); Perandones, (2006); Cabello, (2005). \\
\hline & a: 0,88 & Muñoz Fernández (2016); Augusto-Landa et al. (2012) \\
\hline & a: 0,87 & De la Fuentes et al. (2010). \\
\hline & a: 0,86 & Augusto-Landa et al. (2011 y 2006). \\
\hline & a: 0,60 & Palomera, (2006). \\
\hline & a: 0,90 & $\begin{array}{l}\text { Pertegal et al. (2011); Perandones, (2006); Cabello, (2005); Augusto-Landa et al. } \\
\text { (2011 y 2006). }\end{array}$ \\
\hline \multirow[t]{5}{*}{ CLARIDAD } & $a: 0,88$ & Muñoz Fernández (2016); Augusto-Landa et al. (2012) \\
\hline & a: 0,81 & De la Fuentes et al. (2010). \\
\hline & a: 0,70 & Palomera, (2006). \\
\hline & a: 0.86 & $\begin{array}{l}\text { Muñoz Fernández (2016): Pertegal et al. (2011); Perandones, (2006); Cabello, } \\
\text { (2005): Auqusto-Landa et al. (2011 y 2006) }\end{array}$ \\
\hline & $a: 0,83$ & Palomera, (2006). \\
\hline \multirow[t]{2}{*}{ REPARACIÓN } & $a: 0,80$ & Auqusto-Landa et al. (2012) \\
\hline & $a: 0,76$ & De la Fuente et al. (2010). \\
\hline
\end{tabular}

\section{EQ-i de Bar-On}

El cuestionario EQ-i de BAR-ON (1997, adaptado al castellano por MHS, Toronto, Canadá), evalúa a través de 133 ítems, diferentes habilidades emocionales y sociales. Las respuestas que pueden dar a cada ítem queda recogida en una escala tipo Likert de cinco puntos, que oscila desde "Siempre" hasta "Nunca" (Perandones y Castejón Costa, 2006). Está constituido por cinco dimensiones: intrapersonal, interpersonal, adaptabilidad, manejo del estrés y humor general. Como señala Seagraves-Robinson (2013), de este instrumento podemos encontrar varias versiones: EI EQ-i con 90 ítems (Alavinia y Kurosh, 2012), 51 ítems, llamado EQ-i:S (Pertegal-Felices, Castejón-Costa y Martínez 2011), 38 ítems, llamado EQ-i:YV-O (Fernández, Bermejo, Sainz, Llor, 
Hernández y Soto, 2011) y finalmente de 28 ítems, denominado EQ-i:C (López-Zafra, Pulido y Berrios, 2014). La fiabilidad obtenida con este cuestionario en los distintos estudios considerados se muestra en la tabla 4.

TABLA 4. FIABILIDAD OBTENIDA POR LOS DIFERENTES ESTUDIOS CONSIDERADOS QUE UTILIZAN EL TEST BAR-ON

\begin{tabular}{ccc}
\hline & FIABILIDAD & AUTORES \\
BAR-ON & a: $0,86-0,65$ & Pertegal et al. (2011) \\
EQ-i & a: $0,83-0,69$ & Fernández et al. (2011) \\
\hline
\end{tabular}

\section{WLEIS}

La escala WLEIS no se encuentra asignada en ninguno de los grupos descritos, pero teniendo en cuenta sus características, la hemos considerado en el primer grupo. Esta escala diseñada por Wong y Law (2002), consta de 16 ítems y mide 4 subdimensiones: Percepción intrapersonal (evaluación de las propias emociones), percepción interpersonal (evaluación de las emociones de los demás), asimilación (uso de las emociones) y regulación emocional (regulación de las emociones), (PenaGarrido et at., 2012). En la bibliografía existente, podemos observar diferencias con respecto al uso de la escala de respuesta. Por ejemplo, Pena-Garrido y Extremera (2012), y Rey y Extremera (2011), utilizan una escala Likert de 7 puntos, sin embargo, Atta, Ather y Bano (2013) utilizan una escala Likert de 6 puntos, mientras que Wong, Wong y Peng (2010) de 5 puntos. En la tabla 5 se aprecian la fiabilidad el test total, y cada uno de los parámetros que lo contiene:

TABLA 5. FIABILIDAD OBTENIDA POR LOS DIFERENTES ESTUDIOS CONSIDERADOS QUE UTILIZAN EL WLEIS.

\begin{tabular}{ccc} 
WLEIS & FIABILIDAD & AUTORES \\
PARÁMETROS & a: 0,90 & Rey et al. (2011) \\
AUTORES \\
Fercepción intrapersonal & FIABILIDAD & \\
Percepción interpersonal & a: 0,87 & Pena-Garrido et al. (2012) \\
Asimilación & a: 0,90 & \\
Regulación & a: 0,84 & \\
\hline
\end{tabular}

\section{$C D E-A$}

EL CDE-A se trata de un cuestionario de auto-informe desarrollado por el grupo catalán "GROUP". Divide la educación emocional en 5 dimensiones: cociente, regulación, autonomía emocional, competencias sociales y para la vida y bienestar. Ofrece una puntuación global y otra para cada una de las dimensiones (Pérez-Escoda et al., 2013). El coeficiente de fiabilidad obtenido en los estudios que han usado este cuestionario, puede verse en la tabla 6 . 
TABLA 6. FIABILIDAD OBTENIDA POR LOS DIFERENTES ESTUDIOS CONSIDERADOS QUE UTILIZAN EL CDE-A.

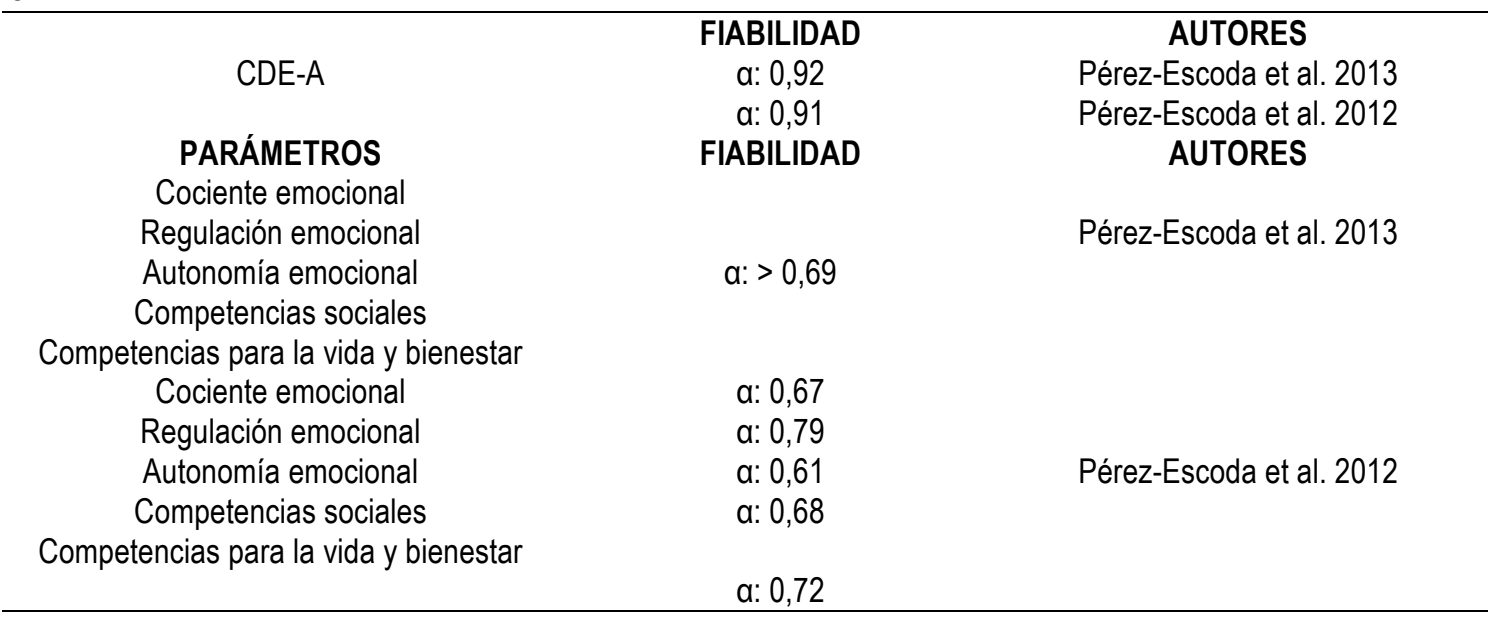

\section{Cuestionario de estrés y competencias emocionales (CECE)}

El cuestionario de estrés y competencias emocionales (CECE) fue diseñado para el estudio de Muñoz de Morales y Bisquerra, (2006). Es un inventario que utiliza medidas de auto-infome formado por 81 ítems y que utiliza un formato de respuesta tipo Likert de 10 puntos. EI CECE que mide tres dimensiones: Bienestar subjetivo, afrontamiento y conciencia emocional. La fiabilidad obtenida por sus autores de este estudio es $\alpha$ : 0,82 (Muñoz de Morales et al., 2006).

\section{Resultados obtenidos en la medición de la IE en docentes}

Para poder hacer una comparativa de resultados, es necesario agrupar los estudios en función a dos criterios:

- Similitud de instrumentos utilizados, y

- Similitud en la interpretación de los resultados.

Cómo se puede observar en las tablas 7 y 8 , aun realizando el estudio con el mismo cuestionario (TMMS), los resultados están expuestos en función a diferentes formas de medida, ya sea "sumatorio de los ítems" (tabla 7) o "utilizando la media" de una escala Likert 1-5 (tabla 8).

\section{TMMS-24 y TMMS}

De los estudios recopilados, ocho miden la IE utilizando el cuestionario TMMS-24 y sólo uno utiliza el TMMS (Extremera et al, 2003). Tras agrupar los estudios con los criterios ya comentados en el párrafo anterior (sumatorio de ítems o realizando la media), proponemos una primera relación de estudios con las investigaciones de Pulido-Martos et al. (2016), Augusto-Landa et al. (2012, 2011), De la Fuente et al. (2010) y Palomera et al. (2006). Estos estudios utilizan el sumatorio de los ítems de cada uno de los parámetros (Atención, Claridad y Reparación) del TMMS-24 (Tabla 7): 
TABLA 7. RESULTADOS OBTENIDOS EN LOS ESTUDIOS CONSIDERADOS QUE UTILIZAN EL TMMS-24 SUMANDO LOS ÍTEMS

\begin{tabular}{cccccc}
\hline Parámetros & $\begin{array}{c}\text { Pulido Martos et } \\
\text { al. }\end{array}$ & $\begin{array}{c}\text { Augusto-Landa et } \\
\text { al. }\end{array}$ & \multicolumn{2}{c}{ De la Fuente et al (*) } & $\begin{array}{c}\text { Palomera et } \\
\text { al. }\end{array}$ \\
\hline ATENCIÓN & $23,3($ sd 6,2$)$ & $23,30($ sd 6,22) & Gr. C: 26,11 & Gr. E: 25,38 & \\
& & & & & 27,95 \\
CLARIDAD & $27,1($ sd 5,5) & $27,06($ sd 5,51) & Gr. C: 25,20 & Gr. E: 26,21 & 24,79 \\
REPARACIÓN & $26,7($ sd 5,3) & $26,71($ sd 5,32) & Gr. C: 26,44 & Gr. E: 25,03 & 27,24 \\
\hline
\end{tabular}

*Programa de Inteligencia Emocional. El resultado expuesto es antes de aplicar el programa.

Otra agrupación de resultados la podemos realizar atendiendo, a si los autores han utilizado la media obtenida en los ítems de cada dimensión (en vez del sumatorio); tal es el caso, de las investigaciones realizadas por Muñoz Fernández (2016), Extremera et al. (2010), Augusto-Landa et al. (2006). Además, hemos incluido en este apartado la investigación de Extremera et al. (2003), puesto que utiliza el TMMS (48 ítems). Los resultados obtenidos para cada una de las dimensiones pueden verse en la tabla 8:

TABLA 8. RESULTADOS OBTENIDOS EN LOS ESTUDIOS QUE UTILIZAN EL TMMS-24 Y TMMS CUANDO SE CONSIDERA LA MEDIA DE ÍTEMS (1-5) OBTENIDA EN CADA DIMENSIÓN.

\begin{tabular}{|c|c|c|c|c|}
\hline Parámetros & $\begin{array}{l}\text { Muñoz Fernández } \\
\text { (2016) }\end{array}$ & $\begin{array}{l}\text { Extremera et al. } \\
(2010)\end{array}$ & $\begin{array}{l}\text { Augusto-Landa et } \\
\text { al. (2006) }\end{array}$ & $\begin{array}{l}\text { Extremera et al. } \\
(2003)\end{array}$ \\
\hline & $3,41$ (sd. 0,76$)$ & $3,33$ (sd. 0,65$)$ & $3,35$ (sd. 0,46$)$ & 3,33 (sd. 0,62 ) \\
\hline & & & & $3,81$ (sd. 0,62$)$ \\
\hline REPARACIÓN & 3,70 (sd. 0,68 ) & 3,62 (sd. 0,62) & 3,23 (sd. 0,43 ) & 3,74 (sd. 0,61 ) \\
\hline
\end{tabular}

Como podemos apreciar, en las tablas 7 y 8 las dimensiones de Claridad y Reparación muestran valores mayores con respecto a la Atención emocional.Los estudios realizados por Perandones et al. (2006) y Cabello et al. (2005) ponen de manifiesto correlaciones de la IE con otras variables, por ello se expondrá en el apartado de "Relaciones con otros constructos"(apartado d).

\section{WLEIS}

Sólo dos estudios midieron la IE con este instrumento. Sin embargo ambos lo utilizan de forma diferente, ya que el estudio de Pena-Garrido et al. (2012) se centra en analizar las dimensiones que tiene esta escala (intrapersonal, interpersonal, asimilación y regulación), y el estudio de Rey et al. (2011) utiliza la puntuación obtenida para el WLEIS total. Para poder hacer una comparativa de resultados, hemos calculado el WLEIS total que habría obtenido Pena-Garrido et al. (2012).

- Pena Garrido et al.: 5,21 (Intra: 5,44; Inter: 5,32; Asim: 5,20; Regu: 4,89;)

- Rey et al.: $\underline{3,34}$

Existe una diferencia importante entre las puntuaciones totales obtenidas en el WLEIS en ambos trabajos. Cabría cuestionarse si tales diferencias, obedecen al tamaño muestral utilizado en cada investigación, o a características propias de las muestras utilizadas: el estudio de Pena Garrido et al. se realizó con una muestra de 245 docentes de Primaria, mientras que el estudio de Rey et al. se llevó a cabo con 123 docentes de Infantil, Primaria y Secundaria.

\section{$E Q-i$ de Bar-On}

Los estudios de Pertegal-Felices et al. (2011) y Fernández et al. (2011) llevan a cabo la medición de la IE en docentes utilizando el cuestionario EQ-i. Sin embargo ambas investigaciones utilizan medidas diferentes: Pertegal-Felices et al. utiliza el EQ-i:S (51 
ítems) mientras que Fernández et al. el EQ-i: YV-O (38 ítems). Los resultados de ambas investigaciones son dispares debido diferencia en la escala Likert, ya que en el cuestionario de 51 ítems, su escala es de 5 puntos, y en el cuestionario de 38 ítems son de 4 puntos. Además, los docentes son de niveles educativos diferentes, secundaria vs. infantil/primaria.

\section{c) Resultados en relación a los programas de intervención docente}

Los tres estudios (ver tabla 1) que han llevado a cabo programas de IE, realizan un diseño cuasi-experimental pretest-postest con un grupo experimental y un grupo control. Los instrumentos utilizados para la medición del constructo IE han sido diferentes en los tres estudios (CDE-A, TMMS-24 y CECE).

El primer estudio seleccionado, (Pérez-Escoda et al. 2013) utiliza el CDE-A. El grupo experimental recibió "formación en educación emocional" en un programa de 30 horas de duración. Trabajaron durante 6 horas cada una de estas dimensiones: 1. Conciencia emocional; 2. Regulación emocional; 3. Autonomía emocional; 4. Competencias sociales; 5 . Competencias para la vida y el bienestar. Los resultados obtenidos indicaban que no existían cambios significativos entre el grupo control y el grupo experimental en las siguientes dimensiones: conciencia emocional, autonomía emocional y competencias de vida y bienestar. Por el contrario si obtuvieron diferencias significativas entre ambos grupos en las dimensiones de regulación emocional y competencia social. Sin embargo, el hallazgo principal de este estudio es la interacción significativa entre Grupo (control y experimental) y Fase (pretest y postest) que demuestra que sólo el grupo experimental incrementa los valores totales de la escala $(p>0,001)$.

El segundo estudio (De la Fuente et al. 2010), sometió al grupo experimental, a un programa de entrenamiento en "autoestima e inteligencia emocional". El programa de entrenamiento integró pautas y elementos del programa de reducción del estrés de Kabat-Zinn (1990), estrategias mindfullness utilizadas en la terapia de aceptación y compromiso (Carrascoso, 2006; Hayes, Strosahl y Wilson, 1999; Wilson y Luciano, 2002), junto con la exposición y debates de metáforas y ejercicios utilizados en dicha terapia, así como varias historias y relatos tomados de la filosofía zen (Deshimaru, 2006) y de la meditación vipassana (Hart, 1994), junto con otras metáforas y ejercicios de elaboración propia. Para la medición del constructo IE utilizaron el TMMS-24. Los resultados hallados ponían de manifiesto que participar en este programa generaba efectos significativos en todas las dimensiones de la IE. Concretamente se encontró, que participar en este programa generaba un incremento positivo del $17,46 \%$ y $35,63 \%$ en claridad y reparación y una reducción del $24,11 \%$ en atención. Es importante comentar, que en este estudio se utilizaron docentes de diferentes niveles educativos; los docentes de secundaria obtuvieron mayores incrementos (pretestpostest) con respecto a los maestros de infantil y primaria.

El último estudio analizado (Muñoz de Morales y Bisquerra, 2006), utilizó un programa de educación emocional (PECERA) con el propósito de prevenir el estrés psicosocial en el aula. La evaluación se centraba en el impacto del programa por parte del profesorado en cada una de las dimensiones del CECE: Conciencia emocional, Afrontamiento y Bienestar subjetivo. Los resultados indicaban que se producía una mejora en la capacidad para identificar y expresar sentimientos en su alumnado (conciencia emocional), mejoraba la regulación emocional en situaciones de ira o frustración e internalización de la norma moral (afrontamiento) y mejoraba las relaciones interpersonales (bienestar subjetivo). 


\section{d) Relación de los parámetros de IE con otros constructos}

En las tablas 9 y 10, se observan las relaciones de las dimensiones de los diferentes cuestionarios (WLEIS y TMMS) que miden la IE con respecto a otros constructos. Las relaciones más frecuentes son las existentes entre la IE y el Burnout (MBI), destacando diferentes correlaciones (positivas y negativas) en función del instrumento utilizado.

TABLA 9. CORRELACIONES ENTRE WLEIS Y OTROS CONSTRUCTOS.

\begin{tabular}{ccccc}
\hline TEST & DIMENSIÓN & CORRELACIÓN & TEST & DIMENSIÓN \\
\hline \multirow{4}{*}{ WLEIS } & & & MBI & Realización personal \\
Vigor \\
& INTRAPERSONAL & & Dedicación \\
& INTERPERSONAL & POSITIVA & UWES & Absorción \\
& ASIMILACIÓN & & & Apoyo social cuantitativo \\
& & & SSQ-6 & Apoyo social cualitativo \\
& REPARACIÓN & & SWLS & Satisfacción vital \\
& & NEGATIVA & MBI & Agotamiento emocional \\
& & & Despersonalización \\
\hline
\end{tabular}

TABLA 10. CORRELACIONES ENTRE TMMS Y OTROS CONSTRUCTO

\begin{tabular}{|c|c|c|c|c|}
\hline TEST & DIMENSIÓN & CORRELACIÓN & TEST & DIMENSIÓN \\
\hline & \multirow{14}{*}{ ATENCIÓN } & \multirow{13}{*}{ POSITIVA } & \multirow{3}{*}{ COPE } & Afrontamiento conductual \\
\hline & & & & Afrontamiento cognitivo \\
\hline & & & & Centrarse en emociones \\
\hline & & & ISE & Sintomatología \\
\hline & & & \multirow{3}{*}{ MBI } & Agotamiento emocional \\
\hline & & & & Despersonalización \\
\hline & & & & Realización personal \\
\hline & & & PANAS & Afecto positivos y negativos \\
\hline & & & RSQ & Respuestas disposicionales \\
\hline & & & \multirow{3}{*}{ SSST } & Supervisión de un superior \\
\hline & & & & Deficiencia \\
\hline & & & & Cooperación \\
\hline & & & WBSI & $\begin{array}{c}\text { Tendencias a suprimir pensamientos } \\
\text { negativos } \\
\end{array}$ \\
\hline & & NEGATIVA & SF-36 & Salud mental y reglas emocionales \\
\hline & \multirow{19}{*}{ CLARIDAD } & \multirow{19}{*}{ POSITIVA } & \multirow{3}{*}{ COPE } & Afrontamiento conductual \\
\hline & & & & Afrontamiento cognitivo \\
\hline & & & & Centrarse en emociones \\
\hline & & & ISE & Sintomatología \\
\hline & & & \multirow{3}{*}{ ISTAS-21 } & Percepción de apoyo social \\
\hline & & & & Calidad de liderazgo \\
\hline & & & & Estima \\
\hline & & & LOT-R & Optimismo \\
\hline \multirow{11}{*}{ TMMS-24 } & & & \multirow{3}{*}{$\mathrm{MBI}$} & Agotamiento emocional \\
\hline & & & & Despersonalización \\
\hline & & & & Realización personal \\
\hline & & & $\mathrm{MH}-5$ & Salud mental \\
\hline & & & PANAS & Afecto positivo \\
\hline & & & $\begin{array}{c}\text { Rosember } \\
\mathrm{g}\end{array}$ & Autoestima \\
\hline & & & \multirow[b]{2}{*}{$\mathrm{S}_{20} / \mathrm{S}_{23}$} & Satisfacción en prestaciones \\
\hline & & & & Satisfacción intrínseca \\
\hline & & & \multirow{3}{*}{ SF-36 } & Función social \\
\hline & & & & Reglas emocionales \\
\hline & & & & Supervisión de un superior \\
\hline
\end{tabular}




\begin{tabular}{|c|c|c|c|c|}
\hline & & & SSST & Punto de vista para realizar mejoras \\
\hline & & & & Bienestar subjetivo \\
\hline & & & SWLS & Satisfacción \\
\hline & & & ISTAS-21 & Percepción de exigencias psicológicas \\
\hline & & NEGATIVA & NEO-FFI & Neuroticismo \\
\hline \multirow{21}{*}{ TMMS-24 } & \multirow{21}{*}{ REPARACIÓN } & \multirow{19}{*}{ POSITIVA } & \multirow[t]{2}{*}{ COPE } & $\begin{array}{c}\text { Afrontamiento centrado en las } \\
\text { emociones }\end{array}$ \\
\hline & & & & Escapa conductual \\
\hline & & & ISE & Sintomatología \\
\hline & & & \multirow{3}{*}{ ISTAS-21 } & Percepción de apoyo social \\
\hline & & & & Calidad de liderazgo \\
\hline & & & & Calidad de estima \\
\hline & & & LOR-R & Optimismo \\
\hline & & & \multirow{3}{*}{$\mathrm{MBI}$} & Agotamiento emocional \\
\hline & & & & Despersonalización \\
\hline & & & & Realización personal \\
\hline & & & $\mathrm{MH}-5$ & Salud mental \\
\hline & & & PANAS & Afecto positivo y negativo \\
\hline & & & $\begin{array}{c}\text { Rosember } \\
\mathrm{g}\end{array}$ & Autoestima \\
\hline & & & \multirow{3}{*}{$\mathrm{S}_{20} / \mathrm{S}_{23}$} & Satisfacción con el ambiente físico \\
\hline & & & & $\begin{array}{l}\text { Satisfacción en las prestaciones e } \\
\text { intrínseca }\end{array}$ \\
\hline & & & & Bienestar subjetivo \\
\hline & & & & Deficiencias y cooperación \\
\hline & & & SSST & Puntos de vistas para realizar mejoras \\
\hline & & & SWLS & Satisfacción \\
\hline & & \multirow[b]{2}{*}{ NEGATIVAS } & NEO-FFI & Neuroticismo \\
\hline & & & RSQ & Respuestas disposicionales \\
\hline
\end{tabular}

\section{Conclusiones}

El presente trabajo ha analizado todos los estudios relacionados con el constructo IE y la docencia en España realizados hasta la actualidad. Tras una revisión bibliográfica, 16 estudios fueron seleccionados tras cumplir el requisito de inclusión "medir la IE en docentes españoles". 2590 docentes con una media de edad de 41,05 años de diferentes niveles educativos, repartidos por todo el territorio español, participaron en pruebas de medición y programas de inteligencia emocional. La participación femenina fue mayor en la mayoría de las investigaciones $(86,67 \%)$ y los estudios se realizaron en mayor medida en los centros educativos públicos, frente a los concertados y privados.

Sin embargo, no se han podido comparar todos los resultados debido a los diferentes instrumentos utilizados, características de los resultados expuestos y diferencias en los niveles educativos por parte del docente (Infantil, Primaria, Secundaria y Universidad).

Con respecto a los instrumentos utilizados, se observa que el cuestionario TMMS-24 es el más utilizado por parte de los investigadores españoles $(57,1 \%)$ debido a la facilidad y brevedad en su aplicación. Entre las conclusiones que nos aportan los estudios que han utilizado los diferentes instrumentos de medidas podemos encontrar:

Cuando se utiliza como instrumento de medida de la IE el TMMS-24 se puede observar, que los docentes que imparten clases en infantil y primaria obtienen mayores puntuaciones (dentro del rango "adecuado") que los docentes de otros niveles educativos (Pertegal-Felices et al. 2011). Además, la claridad y la reparación, 
son mayores que la atención en la mayoría de las investigaciones analizadas. No podemos obtener conclusiones con respecto al sexo ya que los resultados obtenidos nos impiden hacer una valoración por la falta de información de los estudios analizados.

Con respecto a uso de la escala EQ-i de Bar-on, las dimensiones con resultados más óptimos han sido la interpersonal y el humor general cuando se utiliza la versión de la escala de 51 ítems (Pertegal, et al. 2011), mientras que cuando se utiliza la versión de la escala de 38 ítems, las puntuaciones más óptimas se obtienen en la dimensión adaptabilidad y en humor general (Fernández et al. 2011). Este dato puede alentar a futuras investigaciones, que analicen la conveniencia de utilizar una versión u otra.

Las conclusiones más relevantes en relación con la escala WLEIS son las diferencias halladas entre los estudios de Rey et al. (2011) y el de Pena-Garrido et al. (2012). En el primero se informa de la obtención de resultados por debajo de 4 puntos, mientras que en el segundo, los resultados hallados indican puntuaciones por encima de los 5 puntos. Por consiguiente, sería necesario considerar otros datos, que permitan explorar el nivel de IE de los docentes cuando se utiliza este cuestionario. Finalmente, las conclusiones más relevantes de los otros estudios que utilizan otras medidas para determinar la inteligencia emocional (CDE-A y CECE), se centran en la aplicación de programas de IE, con el resultado del aumento considerable de ésta.

Otras conclusiones relevantes son las correlaciones existentes entre las dimensiones del TMMS (atención, claridad y reparación) y el cuestionario WLEIS (intrapersonal, interpersonal, asimilación y regulación) con otros constructos. Así pues, se encuentra que la atención emocional correlaciona positivamente con el afecto emocional, la supresión de pensamientos negativos, el afrontamiento de las emociones, la realización emocional, la disminución de sintomatologías físicas, psíquicas y sociales asociadas al burnout y la disminución de la rumiación, la distracción y la mejora en la solución de los problemas. La claridad y reparación emocional, correlaciona positivamente con el afecto emocional, el afrontamiento conductual y cognitivo del problema, el optimismo, la autoeficacia, la autoestima; además reduce el burnout, potencia el afecto positivo, la satisfacción, el neuroticismo, la responsabilidad, el liderazgo y la calidad mental. Sin embargo, la claridad emocional correlaciona negativamente con la percepción de exigencias psicológicas, y la regulación emocional con el cansancio emocional, la despersonalización y las respuestas disposicionales (menor percepción de exigencias e inseguridad). Por su parte el WLEIS correlaciona positivamente (todas sus dimensiones) con el vigor, la dedicación y la absorción en el trabajo, el apoyo social y la satisfacción vital. Las dimensiones "interpersonal, asimilación y regulación" correlacionan además, con bajos niveles de burnout. Existen correlaciones negativas con el agotamiento y la despersonalización (en la tabla 1 se describen las correlaciones pertenecientes a cada estudio y autor). Los programas de intervención analizados muestran, en general, una mejora en el aspecto emocional, sin embargo es necesario realizar más investigaciones dirigidas a esclarecer esta cuestión. Algunos programas basados en técnicas de mindfullness ayudan a mejorar la claridad y reparación emocional (De la Fuente et al., 2010), y los programas de reducción de estrés en el aula, aumentan la relación estudiante-profesor (Muñoz de Morales y Bisquerra, 2006).

Por todo lo expuesto anteriormente, creemos que el desarrollo de la IE se convierte en un aspecto fundamental en el desarrollo profesional de los docentes. Después de muchas décadas asignando el coeficiente intelectual como un parámetro fundamental a la hora de determinar el éxito y desarrollo en el ámbito laboral, la inteligencia emocional va demostrando su gran importancia. No es de extrañar que la mayoría de 
los estudios analizados, propongan introducir en los programas de formación docente aspectos emocionales para enseñar a los futuros profesores como gestionar sus emociones, y al mismo tiempo dárselo a conocer a sus estudiantes. Desgraciadamente son escasos los programas de inteligencia emocional y por consiguiente la participación docente, aunque su validez haya sido contrastada científicamente (Cabello, Ruiz-Aranda y Fernández-Berrocal, citado por Pérez-Escoda et al. 2013). Creemos por tanto, que sería necesario unificar todas las características que ofrecen cada uno de ellos para diseñar un programa específico, con garantías de éxito y de obligada realización. Se convierte por tanto en condición fundamental, concienciar a los actuales profesores sobre la gestión de sus propias emociones, ya que como han demostrado la mayoría de los estudios, mejora considerablemente la calidad en el entorno laboral.

\section{Referencias}

Abarca, M., Marzo, L. y Sala, J. (2002). La educación emocional en la práctica educativa de primaria. Bordón, 54(4), 505-518.

Aluja, A., Blanch, A. y Biscarri, J. (2002). Síndrome de Quemarse en el trabajo (Burnout) y estrategias de Afrontamiento: Un modelo de relaciones estructurales. Revista de psicología del trabajo y de las Organizaciones, 18(1), 57-74.

Alavinia, P. \& Kurosh, S. (2012). On the Would-be Bonds between Emotional Intelligence and Self-efficacy: The Case of Iranian EFL University Professors. Theory and Practice in Languages Studies, 2(5), 956-964.

Atta, M., Ather, M. \& Bano, M. (2013). Emotional Intelligence and Personality Traits among University Teachers: Relationship and Gender Differences. International Journal of Business and Social Science. 4(17), 253-259.

Augusto Landa, J. M., López-Zafra, E., Berrios-Martos, M. P. \& Pulido-Martos, M. (2012). Analyzing the relations among perceived emotional intelligence, affect balance and burnout. Behavioral Psychology/Psicología Conductual, 20(1), 151-168.

Augusto Landa, J. M., López-Zafra, E., Martínez de Antoñana, R. \& Pulido, M. (2006). Perceived emotional intelligence and life satisfaction among university teachers. Psicothema. 18. 152-157.

Augusto Landa, J. M., López-Zafra, E. y Pulido-Martos, M. (2011). Inteligencia Emocional Percibida y estrategias de afrontamiento al estrés en profesores de enseñanza primaria: propuesta de un modelo explicativo con ecuaciones estructurales (SEM). Revista de Psicología Social, 26(3). 413-425.

Bar-On, R. (2000). Emotional and social intelligence: Insights from the Emotional Quotient Inventory (EQ-i). En R. Bar-On y\& J.D.A Parker (Eds.). The handbook of emotional intelligence. San Francisco: Jossey-Bass. 363-387.

Boyatzis, R. E., Goleman D. \& Rhee, K. (2000). Clustering competence in emotional intelligence: insights from the emotional competence inventory (ECl). En Bar-On, R. \& Parker, J. D. A. (Eds). Handsbook of emotion Intelligence. San Francisco: JosseyBass, 343-362.

Cabello, R., Fernández-Berrocal, P., Ruiz-Aranda, D. y Extremera, N. (2005). Influencia de la inteligencia emocional y estilos de respuestas de factores de riesgo psicosocial en profesores de educación especial. $6^{\circ}$ Congreso Virtual de Psiquiatría. Interpsiquis.
Cabello, R., Ruiz-Aranda, D. y Fernández-Berrocal, P. (2010). Docentes emocionalmente inteligentes. REIFOP, 13(1). 41-49

Cherniss, C., \& Adler, M. (2000). Promoting emotional intelligence in organizations. Alexandria, AV: American Society for Training and Development.

Clavero Muñoz, A. R. (2011). La inteligencia emocional como factor de protección de la violencia escolar. Facultad de Ciencias de la Educación, Universidad de Almería.

De la Fuente Arias, M., Salvador Granados, M. y Franco Justo, C. (2010). Efectos de un programa de entrenamiento en conciencia plena (Mindfulness) en la autoestima y la inteligencia emocional percibidas. Behavioral Psychology / Psicología Conductual, 18 (2). 297-315.

Extremera, N., Durán, A. y Rey, L. (2010). Recursos personales, síndrome de estar quemado por el trabajo y sintomatología asociada al estrés en docentes de enseñanza primaria y secundaria. Ansiedad y Estrés, 16(1). 47-60.

Extremera, N., Durán, A. y Rey, L. (2007). Perceived emotional intelligence and dispositional optimismo-pessimism: Analyzing their role in predicting psychological adjustment among adolescents. Personality and Individual Difference, 42, 1069-1079.

Extremera, N. \& Fernández-Berrocal, P. (2006). Emotional intelligence as predictor of mental, social, and physical healthy in university students. The Spanish Journal of Psychology, 9, 45-51.

Extremera, N. y Fernández-Berrocal, P. (2004). La importancia de desarrollar la inteligencia emocional en el profesorado. Revista Iberoamericana de Educación, 33, 1-10.

Extremera, N., Fernández-Berrocal, P. y Durán Durán, A. (2003). Inteligencia emocional y burnout en profesores. Encuentros en Psicología Social, 1, 260-265.

Extremera, N. y Fernández-Berrocal, P. (2003). La inteligencia emocional en el contexto educativo: Hallazgos científicos de sus efectos en el aula. Revista de Educación, 1(332), 97-116.

Extremera, N. y Fernández-Berrocal, P. (2003). La inteligencia emocional: Métodos de evaluación en el aula. Revista Iberoamericana de Educación, 30, 1-12

Fernández, M. C., Bermejo, R., Sainz, M., Llor, L., Hernández, D. y Soto, G. (2011). Percepción socioemocional de los profesores 
en adolescentes con altas habilidades versus habilidades medias. Revista Electrónica Interuniversitaria de Formación del Profesorado, 14(3). 55-64.

Fernández-Berrocal, P., Berrios-Martos, M. P., Extremera, N. \& Augusto, J.M. (2012). Inteligencia emocional: 22 años de avances empíricos. Behavioral Psychology/Psicología Conductual, 20(1), 197-209

Fernández-Berrocal, P \& Extremera, N. (2002). La inteligencia emocional como una habilidad esencial en la escuela. Revista Iberoamericana de Educación, 29, 1-6.

Fernández-Berrocal, P., Extremera, N. \& Ramos, N. (2004). Validity and reliability of the Spanish modified version of the Trait Meta-Mood Scale. Psychological report, 94, 751-755. doi: 10.2466/pr0.94.3.751-755.

Fernández-Berrocal, P., Alcaide, R., Domínguez Antuñez, E., Fernández McNally, C., Giménez de la Peña, A., Ramos Díaz, N. \& Ravira Espinosa, M. (1998). What is emotional intelligence in a school community? A Spanish high school students's perspective. En ICEL 98 Conference. Findland.

García-Fernández, M. \& Giménez-Mas, S. I. (2010). La inteligencia emocional y sus principales modelos: Propuesta de un modelo integrador. Revista Digital del centro del profesorado Cuevas-Olula (Almería), 3(6), 43-52.

Goleman, D. (1995). Inteligencia emocional. Barcelona: Kairos.

González Robles, A., Peñalver González, J. y Bresó Estevez, E. (2011). La evaluación de la inteligencia emocional: ¿Autoinformes o pruebas de habilidad?. Forum de Reserca, 16, 699-712.

Kabat -Zinn, J. (1990). Full catastrophe living. New York: Dell Publishing.

López-Zafra, E. Pulido, M. \& Berrios, P. (2014). EQI VERSION CORTA (EQIC) Adaptación y validación al español del EQ-i en universitarios. Boletín de psicología, 110, 21-36.

Matthews, G., Zeidner, M. y Roberts, R. D. (2002). Emotional intelligence: Science and myth. Boston: MIT Press.

Mayer, J. \& Cobb, C. (2000). Emotional policy on Emotional Intelligence: does it makes sense?. Educational Psychology Review, 12, 163-183.

Mayer, J., Salovey, P. \& Caruso, D. (2000). Emotional intelligence as Zeitgeist, as personality, and mental ability. En R. J. Sternberg (Ed.), Handbook of emotional intelligence .San Francisco: Jossey Bass. 92-117.

Mayer, J. \& Salovey, P. (1997). What is emotional intelligence? En P. Salovey \& D. Sluyter (eds): Emotional developement and emotional intelligence: implication for educador. New York, Basic Books.

Mayer, J., Caruso, D. \& Salovey, P. (1999). Emotional intelligence meets traditional standards for an intelligence. Intelligence, 27. 267-298.

Mearns, J. y Cain, J. E. (2003). Relationship between teachers' occupational stress and their burnout and distress: roles of doping and negative mood regulation expectancies. Anxiety, Stress and Coping, 16, 71-82.
Mestre, J.M., Guil, R., Lopes, P. N., Salovey, P. \& Gil-Olarte, P. (2006). Emotional Intelligence and social and academic adaptation to school. Psicothema, 18, 112-117.

Muñoz de Morales lbáñez, M. y Bisquerra Alzina, R. (2006). Evaluación de un programa de educación emocional para la prevención del estrés psicosocial en el contexto del aula. Ansiedad y Estrés 12(2-3), 401-412.

Muñoz Fernández, M.L (2016). Inteligencia emocional percibida y su relación con la satisfacción y el bienestar subjetivo del profesorado. Inteligencia emocional y bienestar II. 660-675. Madrid: Editorial la UNE

Palomera Martín, R., Gil-Olarte Márquez, P. A. y Brackett, M. (2006). ¿Se perciben con inteligencia emocional los docentes? Posibles consecuencias sobre la calidad educativa. Revista de Educación, 34. 687-703.

Palomera, R., Fernández-Berrocal, P. y Brackett, M. A. (2008). La inteligencia emocional como una competencia básica en la formación inicial de los docentes: algunas evidencias. Revista Electrónica de Investigación Psicoeducativa, 6(15), 437-454.

Parker, J. D. A., Summerfeldt, L. J., Hogan, M. J. \& Majeski, S. A. (2004). Emotional intelligence and academia sucess: examining the transition from high school to university. Personality and Individual Differences, 36(1), 163-172.

Pena Garrido, M. y Extremera Pacheco, N. (2012). Inteligencia emocional percibida en profesorado de primaria y su relación con los niveles de burnout e ilusión por el trabajo (engagement). Revista de Educación, 359. 604-627.

Perandones González, T. M. y Castejón Costa, J. L. (2006). Estudio correlacional entre personalidad, inteligencia emocional y auto eficacia en profesorado de educación secundaria y bachillerato. V Congreso Internacional "Educación y Sociedad".

Bisquerra, R.; Alegre, A.; Pérez-Escoda, N.; Filella, G.; (2012). Desarrollo de la competencia emocional de maestros y alumnos en contextos escolares. Electronic Journal of Research in Educational Psychology, 124, 1183-1208.

Pertegal-Felices, M. L., Castejón-Costa, J. L. y Martínez, M. A (2011). Competencias socioemocionales en el desarrollo profesional del maestro. Educación XXI, 14(2), 237-260.

Pulido-Martos, M. López-Zafra, E. Estévez-López, F. \& AugustoLanda, J.M. (2016). The Moderator Role of Perceived Emotional Intelligence in the Relationship between sources of stress and Mental Health in Teacher. The Spanish Journal of Psychology, 19(7), 1-10.

Rey, L. y Extremera, N. (2011). El apoyo social como un mediador de la Inteligencia Emocional percibida y la satisfacción vital de una muestra de profesorado. Revista de Psicología Social, 26(3). 401412.

Sala, F. (2002). Emotional competence inventory: Technical manual. Philadelphia, PA: McClelland Center For Research, HayGroup.

Salovey, P. \& Mayer, J. D. (1990). Emotional intelligence. Imagination, Cognition, and Personality, 9, 185-211. 
Schutte, N. S., Malouff, J., Simunek, M., Hollander, S. \& Mckenley, J. (2002). Characteristic emotional intelligence and emotional wellbeing. Cognition and Emotion, 16, 769-785.

Shutte, N. S., Malouff, J. M., Hall, L. E., Haggerty, D. J., Cooper, J. T., Golden, C. J. \& Dornheim, L. (1998). Development and validation of a measure of emotional intelligence. Personality and Invididual Differences. 25, 167-177.

Seagraves-Robinson, T. Y. (2013) The relationship between Florida's beginning elementary teachers' Emotional Intelligence, select demographic characteristics and performance. Florida agricultural and mechanical university.

Sutton, R. \& Wheatley, K. (2003). "Teachers"e emotions and teaching: A review of the literature and directions for future research. Educational Psychology Review, 15, 327- 358.
Valles, A. y Vallés, C. (2003). Psicopedagogía de la Inteligencia Emocional. Valencia: Editorial Promolibro.

Wilson, K. G. y Luciano, C. (2002). Terapia de aceptación y Compromiso: Un tratamiento conductual orientado a los valores. Madrid: Editorial Pirámide.

Wong, C. \& Law, K. S. (2002). The effects of leader and follower emotional intelligence on performance and attitude: An exploratory study. The Leadership Quarterly, 13, 243-274.

Wong, C., Wong P. \& Peng, K. (2010). Effect of Middle-level Leader and Teacher Emotional Intelligence on School Teachers' Job Satisfaction: The Case of Hong Kong. Educational Management Administration \& Leadership, 38(1), 59-70. 